\title{
Performance and Applications of Gallium-Nitride Monolithic Microwave Integrated Circuits (GaN MMICs)
}

\author{
Jonathan B. Scott ${ }^{a}$ and Anthony E. Parker ${ }^{b}$ \\ ${ }^{a}$ Waikato University, Hamilton, New Zealand; \\ ${ }^{b}$ Macquarie University, Sydney, Australia
}

\begin{abstract}
The evolution of wide-bandgap semiconductor transistor technology is placed in historical context with other active device technologies. The relative rapidity of GaN transistor development is noted and is attributed to the great parallel activity in the lighting sector and the historical experience and business model from the III-V compound semiconductor sector. The physical performance expectations for wide-bandgap technologies such as Gallium-Nitride Field-Effect Transistors (GaN FETs) are reviewed. We present some device characteristics. Challenges met in characterising, and prospects for modeling GaN FETs are described. Reliability is identified as the final remaining hurdle facing would-be foundries. Evolutionary and unsurprising applications as well as novel and revolutionary applications are suggested. Novel applications include wholly monolithic switchmode power supplies, simplified tools for ablation and diathermy in tissue, and very wide dynamic range circuits for audio or low phase noise signal generation. We conclude that now is the time to embark on circuit design of MMICs in wide-bandgap technology. The potential for fabless design groups to capitalise upon design IP without strong geopraphic advantage is noted.
\end{abstract}

Keywords: Gallium Nitride, Wide-bandgap, MMIC, Compound semiconductor, circuit design

\section{HISTORY}

Vacuum tubes ("valves") held sway for 50 years in the field of electronics. They are used to this day, a century after their invention, in several narrow niches including studio microphone preamplifiers, enthusiasts' high-fidelity sound systems, and very high power-bandwidth RF amplifiers. Figure 1 presents a pictoral chronology of current active device technologies. Consider that the humble vacuum tube was relatively easy to understand in terms of the physics governing its operation, relatively easy to construct, and it enabled the electronics revolution, bringing us radio communication, sensitive measurement, precise control of electrical and mechanical processes, and broadcast entertainment, including television. Its star rose over a period of 50 years, and its area of play declined to a niche in about 20 years once the next step had been realised.

Half way through the reign of tubes the FET was patented (in 1934) but it could not be made. Eventually the BJT was invented and constructed. In the 1950s transistors appeared, and they were made with Germanium. Readers over 50 years old may have started tinkering with transistors such as the AC126 and AC127 that were common in the 1960s.

Silicon formed a better material system. Around a decade after the Germanium device was made Silicon followed, and within a few years the Silicon BJT surpassed the Germanium version. Germanium offered a lower forward voltage drop and so it held on until the late 1980s at which point the last Germanuim manufacturer disappeared. The Silicon family, if we may group Silicon and Germanium into a family based on their presence as valence-four atoms in the fourteenth group of the periodic table, rose to preeminence in far less time than tubes. This rise was driven simply by the potential for improved robustness, reduced power consumption, and reduced size, not by the vast panoply of applications that appeared with the move to planarity through the development of photolithographic manufacture and then the integrated circuit. By any measure this was a rapid development.

Private Bag 3105, Hamilton, 3240, New Zealand Telephone: +64(7)838-4909, E-mail: jbs@waikato.ac.nz 




Figure 1. A pictoral summary of electronic developments and the active device technologies that were used throughout the electronics era. Note that new developments tend to use the latest technology family. Germanium alone has effectively disappeared, as it was eventually surpassed in every aspect, although it is contributes now in the Silicon system as a means to produce a compound-semiconductor heterojunction in SiGe devices.

Meanwhile, physicists had been speaking of better solid-state material systems. The one that caught the attention of observers mainly in the USA was the "III-V" system, epitomised by Gallium-Arsenide, GaAs. Large sums of money from the Defence Advanced Research Projects Agency, DARPA, lubricated two advances in semiconductor device technology. The first was the planarity-enabled move to VLSI, where the challenges layand continue to lie - in the size of the photolithographic features and the immense complexity of circuits that must be handled as a black box. The second advance, and the one of interest to us here, was the move to potentially better material systems, the move into the compound semiconductor technology GaAs.

The compound semiconductor material formed of crystals of Gallium and Arsenic offered advantages over Silicon: The intrinsic mobility of charge carriers was about 8 times larger, so device speeds would potentially be much greater; the substrate was relatively insulating with a large dielectric constant, so that electromagnetic passives such as transmission lines and inductors could be fabricated along with transistors, resistors and capacitors; also the bandgap was larger, so visible-light emission was possible. With the DARPA-driven projects, the naturally more difficult compound semiconductor industry came into existence. At the time great effort was invested in telling engineers about the new semiconductor technology revolution, and the IEEE even produced a series of videotapes to carry the message.

Circa 1980 GaAs-based devices took the production electronics speed record, offering the highest $F_{t}$, and 
they also achieved the lowest noise figure, and enabled the fastest logic gates. This was the era of satellite TV and the Cray supercomputer. This was also the era of the Light-Emitting Diode, the LED. It is not so easy to make GaAs circuits. The chemistry - more like alchemy even today - is more involved, from the deposition of compound semiconductor to the etching. It seems unlikely that GaAs will ever get cheaper to manufacture for these reasons. However, its intrinsically higher mobility more than offsets its lousier thermal conductivity, and GaAs is found in many demanding niche applications. It is likely that the power amplifier in your mobile phone is a III-V-based circuit, and your broadband connection would not be either so broad or so cheap were it not for GaAs.*

By the late 1990s the "Indium Phosphide" variant of the III-V family had come to the fore, particularly for Heterojunction Bipolar Transistors, HBTs. Ternary and quaternary layers, sometimes carefully graded ${ }^{\dagger}$ are a particular feature. The InP family is really an extension of GaAs, as it is a III-V semiconductor family where Indium and Phosphorus join Gallium and Arsenic. As such it does not warrant a separate "group" in figure 1, though we show two distinct lines in the III-V group.

The III-V family took less time to reach commercial viability than had Silicon. In spite of its greater difficulty, greater cost, and niche future, it was pretty much fully established in 20 years. In that time the mainstream devices included MESFETs, HBTs and HEMTs. The rise of GaAs differed from the rise in Germanium and Silicon in that it was not targeted to replace the previous generation, but to enable new achievements.

By 2000 wide-bandgap devices had been fabricated, and people were speaking of the speed with which progress was being made in the "wide-bandgap technologies". The main device to date is the equivalent of the humble and original GaAs MESFET. It is fabricated with a Gallium-Nitride mix on either a Silicon substrate or a Silicon-Carbide substrate. Within 5 years foundry services touting GaN technology appeared, and at the time of writing foundries are actively competing for customers. In the 1990s circuit designers sought foundries for Si and III-V fab, but in 2007 foundries are seeking customers. This fast maturation is a business problem.

The authors attribute the relative rapidity of the development of GaN to two factors:

- Parallel work using wide-bandgap semiconductor to address the lighting market with solid-state, white emitters; and

- the existence of a technology and business model, in the form of the III-V community, for the rise of a new technology star.

However, the cause of GaN's rapid rise is not nearly as interesting as the potential impact of the technology, and the extent to which this has not yet been realised.

\section{PRACTICAL WIDE BANDGAP TECHNOLOGY}

What does wide bandgap offer? The most compelling attribute seen to date is probably the availability of blue and white light-emitting diodes. The story of Nakamura and Nichia is now the stuff of semiconductor folklore. The wide-bandgap semiconductor fabrication infrastructure has been paid for by the blue laser diode and white LED markets that followed Nakamura's success. Solid-state light from the laser in your DVD drive to the LEDs in your flashlight and your solar garden lights depends upon wide-bandgap technology. After all the years of operation GaAs LEDs remain substantially confined to electronic indicators and traffic lights (or at least one third of them) but white ones have become ubiquitous.

Our interest, however, is in transistors fabricated in this technology, and in integrated circuits built around these transistors. Such ICs that include passive components and are intended for high-frequency applications are

\footnotetext{
*It is worth noting here that it is the high power-bandwidth product that is of interest, and if your mobile phone uses an InP-based power amplifier it is at least as much for its low power consumption as its potential maximum bandwidth that it has been used over a Silicon circuit. This trade-off explains why Silicon can boast matching speeds in so many publications yet does not at once capture the cellphone PA market.

†The process of "grading" means varying the mix of the alloy continuously through a layer.
} 
usually referred to as MMICs, for Microwave Monolithic Integrated Circuits, to distinguish technologies required to support transmission lines and inductors as well as transistors, resistors and capacitors.

There has appeared an enormous literature on GaN transistors. We do not intend to duplicate any of this here. We will not discuss the solid-state physics nor the fabrication details. Articles have appeared in Spectrum giving glorious summaries of the prospects. Readers are referred to articles such as. ${ }^{1}$ In such articles a small number of key points are identified:

- Wide-bandgap transistors in GaN offer 10 times the power-bandwidth of GaAs ones;

- On a $\mathrm{SiC}$ substrate, they have better thermal conductivity than Silicon;

- Breakdown voltage can be ten times that of Silicon;

- They will happily operate 150C above where silicon transistors stop working.

These are generalisations, as many device properties can be traded off against others, but for comparable situations in other ways, these advantages can be expected.

GaN transistors are currently available or are promised by a number of companies. These include Nitronix, HRL, Teledyne (Rockwell), MicroGaN, TriQuint, RFMD, Northrup-Grumman, Fujitsu/Eudyna, BAE, Qinetiq, and Cree, but more are reported to be coming. The number offering foundry services is reported to have increased $26 \%$ since 2003. Strategies Unlimited reports that no less than 232 companies were participating in GaN production as of early 2005, and that the GaN production market will reach US $\$ 7.6$ billion by 2009, although this is presumed to be mostly lighting-related.

If this boom is "for real", how come we do not see GaN transistors in the local electronics stores as commonly as we see blue LEDs? Before trying to answer this, let us look at some actual GaN device characteristics.

\subsection{Device Characteristics}

There emerge two approaches to GaN transistors. One involves a SiC substrate which is very expensive, and the seond involves using a silicon wafer with some proprietary method of interface to the GaN epitaxial layer. In general, less cost-sensitive or more demanding applications will look to a $\mathrm{SiC}$ substrate, while volume or less-demanding applications take advantages of the silicon approach. There should be no ultimate difference in the epitaxial design between the two, but the division makes business sense. Passives should also be superior on the full $\mathrm{SiC}$ substrates.

Figure 2 shows a typical drain characteristics of a microwave GaN FET fabricated on a Silicon substrate. This particlular device was measured by one of us some three years ago. The characteristics were measured on-wafer, so in order not to melt the device the data was measured under pulsed conditions. Operating at full power on-wafer is not possible with such high-power devices as they cannot be effectively mounted on a heat sink.

Working with high-power GaN devices presents unique challenges. Firstly, for devices that are on $\mathrm{SiC}$ rather than silicon, the wafer, having a high bandgap, appears quite transparent. This can make landing probes under a microscope an interesting experience.

Because the devices are truly microwave transistors, they readily oscillate if not suitable terminated, and so must be probed with microwave probes, typically GSG coplanar probes of the type supplied by PicoProbe or Cascade. These probes cost from US $\$ 500$ upwards. If the device fails, the probe tips can be vaporised with less power than the transistor was dissipating, another incentive to use pulsed operation. It is sobering to observe that a GaN transistor failing on-wafer can be accompanied by a flash and a sound audible across a large laboratory!

The data of Figure 2 can seem rather unremarkable. For this reason we present the data in figure 3. A similar transistor to the one appearing in figure 2 has had its characteristics superimposed upon those of a "high power" GaAs MESFET of comparable size and frequency response. The GaAs MESFET process in question is typical of those responsible for some of the highest power travelling-wave amplifiers made by the likes of Centellax, Agilent 


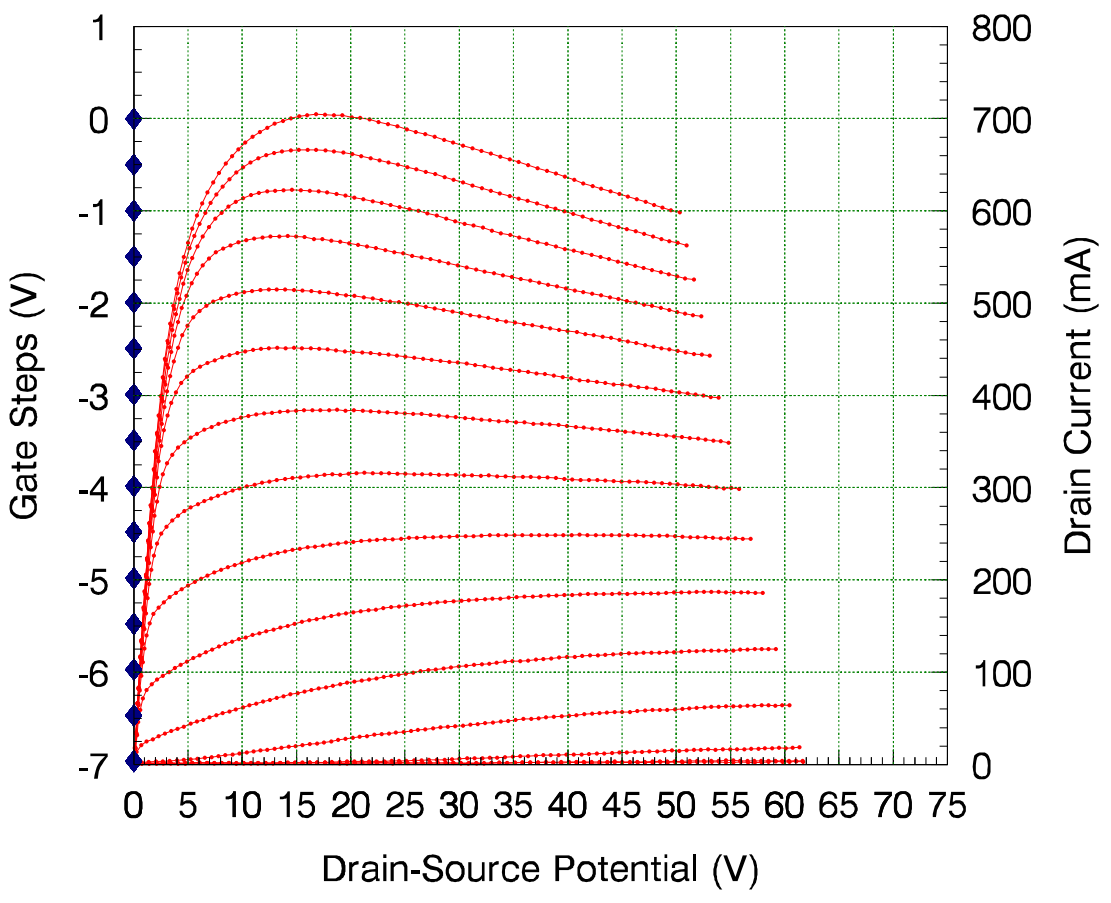

Figure 2. Characteristics of a $1 \mathrm{~mm}$ GaN FET. The subject device was fabricated at MicroGaN circa 2004. The data shown here was measured on wafer, and under pulsed conditions in order to prevent destruction of the device.

Technologies and Avago. Note that the working $F_{t}$ of the GaN device is actually higher than that of the GaAs MESFET. Figure 4 plots measured device $F_{t}$ against drain current and applied voltage.

The data presentation in figure 4 may be unfamiliar. The device S-parameters are measured across its entire operating range, and then characteristics are extracted and plotted as a 3-dimensional surface against relevant device parameters. This $3 \mathrm{D}-F_{t}$ plot conveys to the trained eye a number of interesting aspects of the device. First, note the peak $F_{t}$. This is the single number often quoted in papers reporting new devices. Sometimes the peak is very narrow, but in the case of this device the peak is broad, as indicated by the contour plot superimposed on the I-V plane. A broad peak such as this indicates a very usable device, where biassing will not be critical. Also, the surface is relatively smooth throughout the desirable operating range. If this is not the case, a device is apt to introduce additional signal distortion when processing high-frequency signals, or in other words the dc and pulsed device characteristics will form a bad predictor of operating linearity. In every way, figure 4 is pleasing to the eye of the circuit designer.

GaN FET characteristics are not seriously affected by temperature. (We do not present proof of this here in the interests of brevity as it is well-proven in the literature.) GaN FETs do not show the severe memory effects for signals in modulation bandwidths that are the hallmark of GaAs FETs. ${ }^{\ddagger}$ GaN FETs can be made very stable and free from oscillations. Figure 5 gives examples of measurements carried out on the same FET as measured in figures 2 and 4 .

A powerful method of putting GaN capability in context is to consider what can be achieved in broadband amplifiers. To an extent, the power delivered by a matched amplifier can be arbitrarily extended by adding

\footnotetext{
${ }^{\ddagger}$ GaN FETs do exhibit trapping effects and this is expected from our theoretical understanding. However, these phenomena can readily be confined to near-dc frequencies, and can be expected not to present the same problems to designers as do the III-V devices. Thus the conventional schemes to search for memory effects tend to give GaN FETs a clean bill of health.
} 


\section{Scaled FET Comparison}

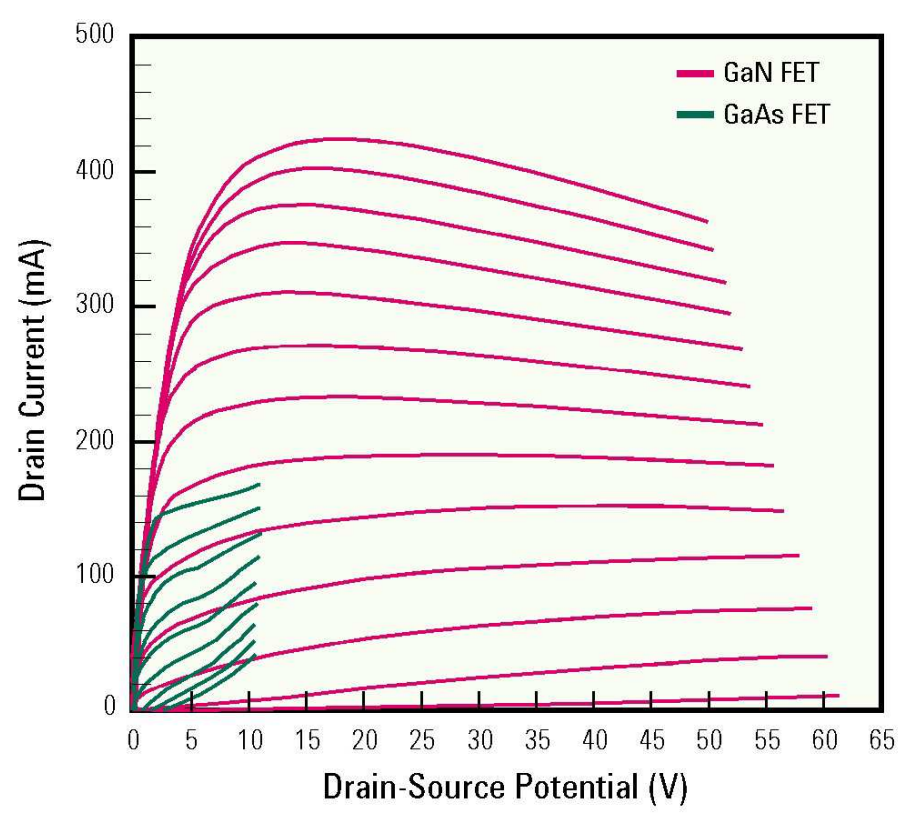

Figure 3. Characteristics of a GaAs MESFET with $F_{t} \approx 25 \mathrm{GHz}$ superimposed on those of a GaN FET similar to the one whose characteristics appear in figure 2 and whose $F_{t}$ is plotted in figure 4 . The gate width of the GaN device is actually slightly smaller than that of the MESFET.

more transistor width and appropriately redesigning the matching network. This is not possible in a broadband design, and solid-state travelling-wave amplifiers (TWAs) are the height of this design. ${ }^{3}$ Figure 6 projects on the power-bandwidth plane the regions currently served by III-V-based TWAs, and indicates our projections for what will be possible in GaN. The original of this diagram showed a number of specific devices but that information remains proprietary, so we present the results without the specific data.

The projections in figure 6 are based on measurements we have taken on actual devices, as well as published data. Devices with $12-30 \mathrm{GHz} F_{t}$ on silicon substrates and devices with $40-90 \mathrm{GHz} F t$ on SiC have been used in rudimentary TWA designs. This data is partucularly reliable in the cases where we were knew details of devices as well as associated TWA designs as was the case for several TWA circuits manufactured and maintained by Agilent Technologies, where these calculations were done. We believe that single-circuit TWAs with 5 Watt capability and $\mathrm{dc}-10 \mathrm{GHz}$ bandwidth are possible with existing silicon-based devices, while $23 \mathrm{dBm}$ from low frequency to $50 \mathrm{GHz}$ is possible with the current generation of SiC-based devices. These figures are significantly greater than currently-available MMICs.

\subsection{Reliability}

Reliability remains an issue in GaAs CS (Compound Semiconductor) technology, and the whole III-V CS model revolves around achievement of appropriate reliability. Accelerated life testing and successful results from this are key to a foundry's business success. Reliability is the most-often cited reason for reticence on the part of individuals or companies whose application could benefit from a move to GaN.

In the late 1990s the MTTF of GaN devices in laboratory conditions was measured in hours, not years. Major GaN fab facilities maintain a roadmap of reliability. Sadly this information is most closely guarded, and frequently published only when it is favourable. Nevertheless, publications are starting to include reliability data.

However, it seems that nobody in the industry doubts that the reliability will be achieved. It is being achieved for LEDs in the same material system, and foundries will quote the calendar year at which they expect 


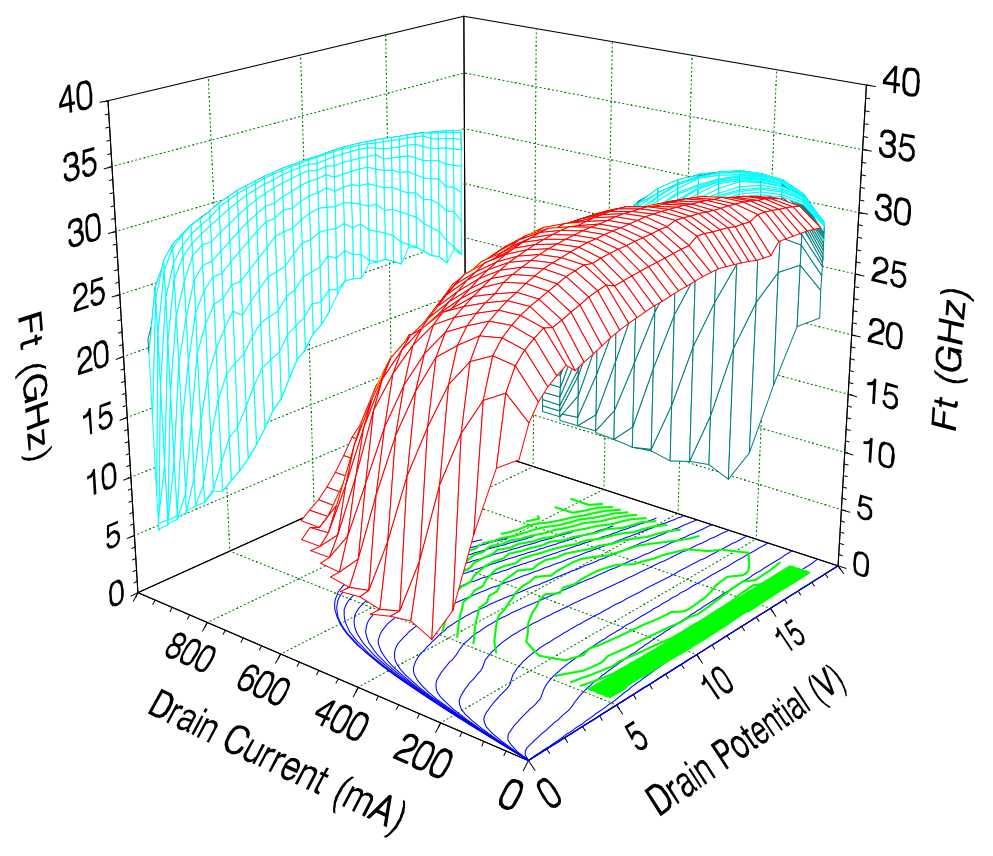

Figure 4. Plot of the $F_{t}$ of a GaN FET as a function of drain current and drain voltage presented as a 3-dimensional surface. Drain characteristics are shown on the lower x-y (I-V) plane, with a contour plot of the $F_{t}$ superimposed on top. Note the relatively broad region where the $F_{t}$ is maximised, implying straightforward use and good linearity.

to achieve "commercial rel". It is thus our contention that the reliability argument is a good one for not moving to commercial application, but it is not a good argument for postponing the circuit research and application development. With a two-year headstart a company can capture a market, and this is what will be available if the design is undertaken now.

\section{APPLICATIONS}

The volume of publications on Field-Effect Transistors in the Gallium-Nitride system (GaN FETs) has increased dramatically since the turn of the millennium, yet much remains speculative with regard to applications. ${ }^{11}$ Large $\mathrm{R} \& \mathrm{D}$ concerns are concentrating on applications of GaN in mobile communications and radar. Their approach is understandable, since these areas have a demonstrated need for the capability of GaN, and offer good probability of providing prompt return on investments in GaN technology. We think of these as "no brainer" applications.

In a popular artcle it has been stated that "None of this is to suggest that gallium nitride will be the next silicon. But it just might be the next gallium arsenide". 1 This may well be true, but we will suggest that GaN will enable circuit applications that are beyond any current active device. We believe that there is considerable room for GaN-based circuits in niche applications, either currently ill-served by Silicon and III-V technologies, or even in markets with no existing semiconductor penetration. ${ }^{7,8,10}$

Much GaN foundry availability is a result of a set of DARPA (Defense Advanced Research Program Agency) contracts let in 2005, and competitive response in Europe and Japan to that investment. The DARPA contracts aim "to transition gallium nitride technology from development to production", as previously happened with Gallium Arsenide (GaAs) and Indium Phosphide (InP) technologies. These DARPA contracts end in 2008, and some carry a requirement that foundry services be offered to enable industry to benefit. 

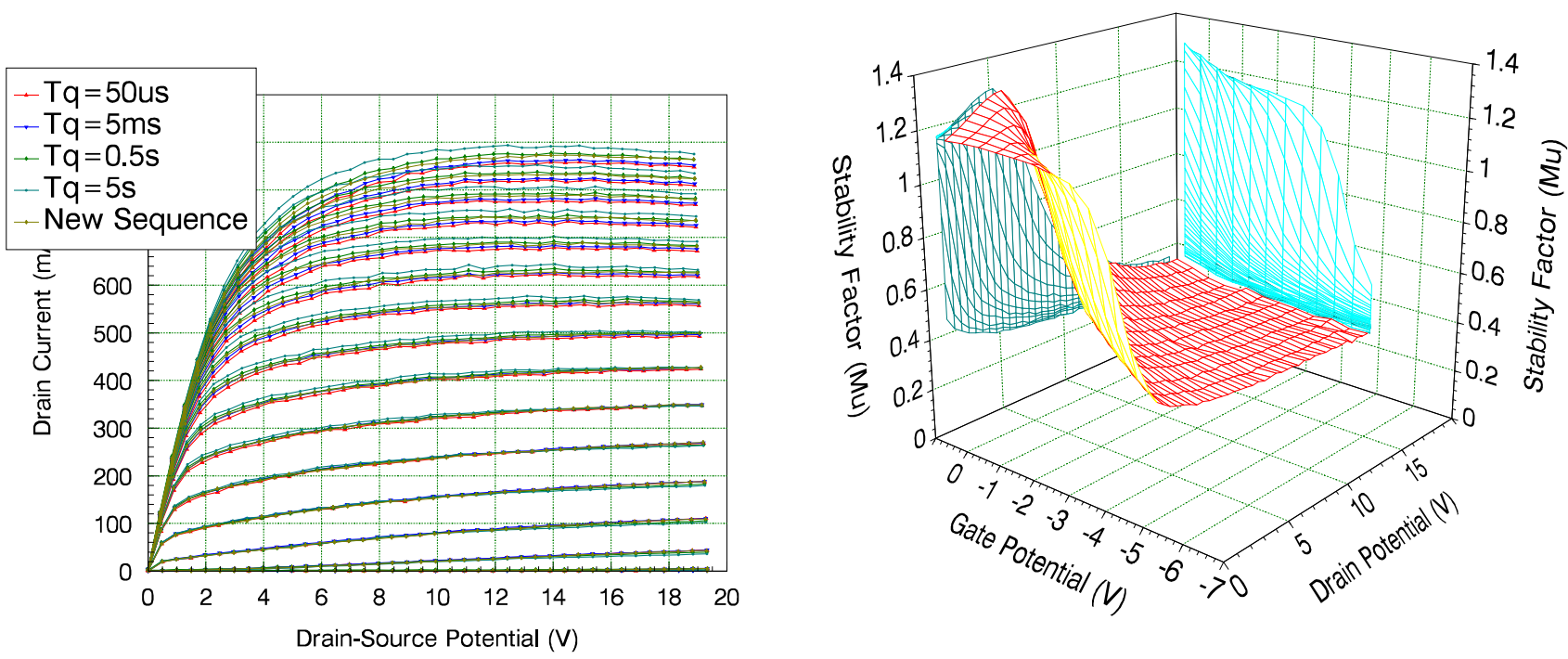

Figure 5. Plots of (a) pulsed characteristics taken with widely varying quiescent interval and pseudo-random point order to expose memory effects and (b) stability factor as a function of drain bias to expose potentially unstable regions. Experienced readers will note that the traces in graph (a) are relatively smooth and do not change much with quiescent interval, indicating a healthy absence of memory effects with time constants near modulation frequencies. Readers will similarly note that Rollet's stability factor is below 1 for most of the active-bias region indicating gain and bandwidth enough to make the device potentially unstable but simultaneously capable of useful operation. The stability surface is smooth and well-behaved.

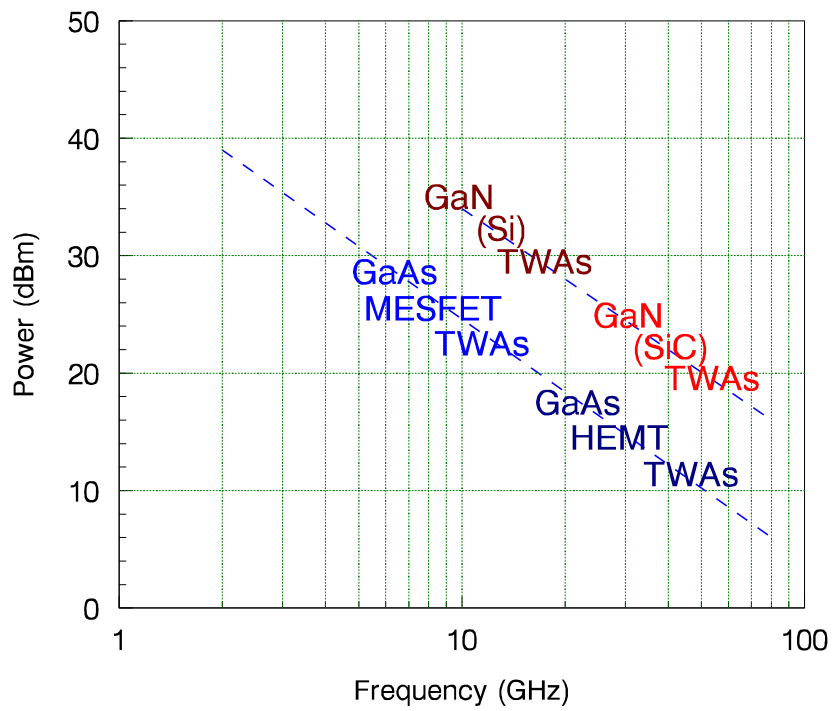

Figure 6. A map showing the power-bandwidth regions served by current deigns in GaAs and predicted to be served by GaN for wide-bandwidth power amplifiers such as TWAs. 
Modeling of transistors to enable the simulation of circuits prior to fabrication is a critical step in the progress of a new semiconductor technology. The availability of CAD models signals the readiness of a technology for practical application. Typically equivalent-circuit models appear, followed by large-signal models of increasing sophistication. In the case of GaN transistors, both small-signal equivalent circuit models ${ }^{12}$ and initial largesignal models including dispersion effects ${ }^{13}$ have appeared in the literature.

In order to make our point, we make a case for several original circuit applications for GaN MMICs.

\subsection{High-Power Oscillator for Medical Diathermy}

Diathermy and ablation are regular medical procedures that involve the delivery of heat by one means or another. When this heating must be local, high-frequency or microwave equpment can be used, or direct dc power dissipation. In the high-frequency case, the equipment consists of a power amplifier, an umbilical cable, and a small antenna.

The vision for GaN-based diathermy tools is to replace the current "microwave antenna on a length of thin coax" 23,24 with a monolothic dc-microwave oscillator based on a GaN MMIC. The high-voltage capability will mean modest supply current, and only a low-fidelity oscillator will be required. The power will be supplied to the probe tip at dc, giving low loss, low cost, and fine mechanical form. Freed from the need to deliver power through coax, the RF energy can be delivered at high gigahertz frequencies, giving highly localised heating.

The rugged nature of GaN makes it perfect for operation in a hostile environment, at elevated temperatures. This application has the added bonus that if the ICs were to be cheap enough, they would be a "disposable" component much like the blade of a scalpel, discarded after use rather than sterilised. In such an application, all reliability concerns vanish!

\subsection{High-Voltage Oscillator for Low Phase Noise}

The vision for low phase-noise oscillators rests on the expectation that the high-voltage, low-noise capability of GaN will give it a wider dynamic range than any previous solid-state technology. ${ }^{11,14,17}$ While the noise floor may rise a $\mathrm{dB}$ or two, the maximum output signal will rise by a much larger amount. The noise performance of current GaN FETs is not far from expectation. Figure 7 presents measurements of the same simple $1 \mathrm{~mm}$ GaN FET biassed at points selected for high-voltage and high-power operation. These FETs, not aimed at such an application, should give something like 8dB improvement compared to a GaAs version.

If the device is optimised, or a suitable process becomes available, bandwidth can be traded off for increased breakdown voltage. In this case, we might see many more dB of improvement in the dynamic range.

Low phase-noise oscillators differ from the oscillators with diathermy application in needing a high-Q resonator. In the past, the power tolerance of the resonator has been competing with semiconductor technology as the upper dynamic range limit, as the resonators suffer mechanical failure if driven too hard, just like a quartz crystal. However, high-voltage dielectric resonators are now available. ${ }^{26}$

\subsection{Monolithic Switchmode Power Conversion}

The last 20 years has seen SwitchMode Power Supplies (SMPS) shrink in cost and size as operating frequency increased. The increased frequency has allowed the magnetics to become ever smaller and lighter, since less energy must be pumped across the converter in each cycle, the more often a conversion cycle occurs. There are also advances in design, including resonant converters, employing natural circuit oscillations to improve efficiency. Resonance in particular has loosened the constraint that the active device switch rapidly compared to the cycle frequency in order to minimise loss.

Our vision for GaN-based switchmode systems is to integrate the entire converter in an IC using GaN to implement converters at $\mathrm{GHz}$ frequencies. ${ }^{20,21}$ The converter would employ the bandwidth available from a GaN device to accomodate design with very small inductance on-chip, and great tolerance to variations in interconnect inductance. There has never been a demonstration of wholly monolithic SMPS, since silicon and GaAs MMICs cannot offer the breakdown voltages simultaneously with fast enough transistors - they have too low a power-bandwidth product. 


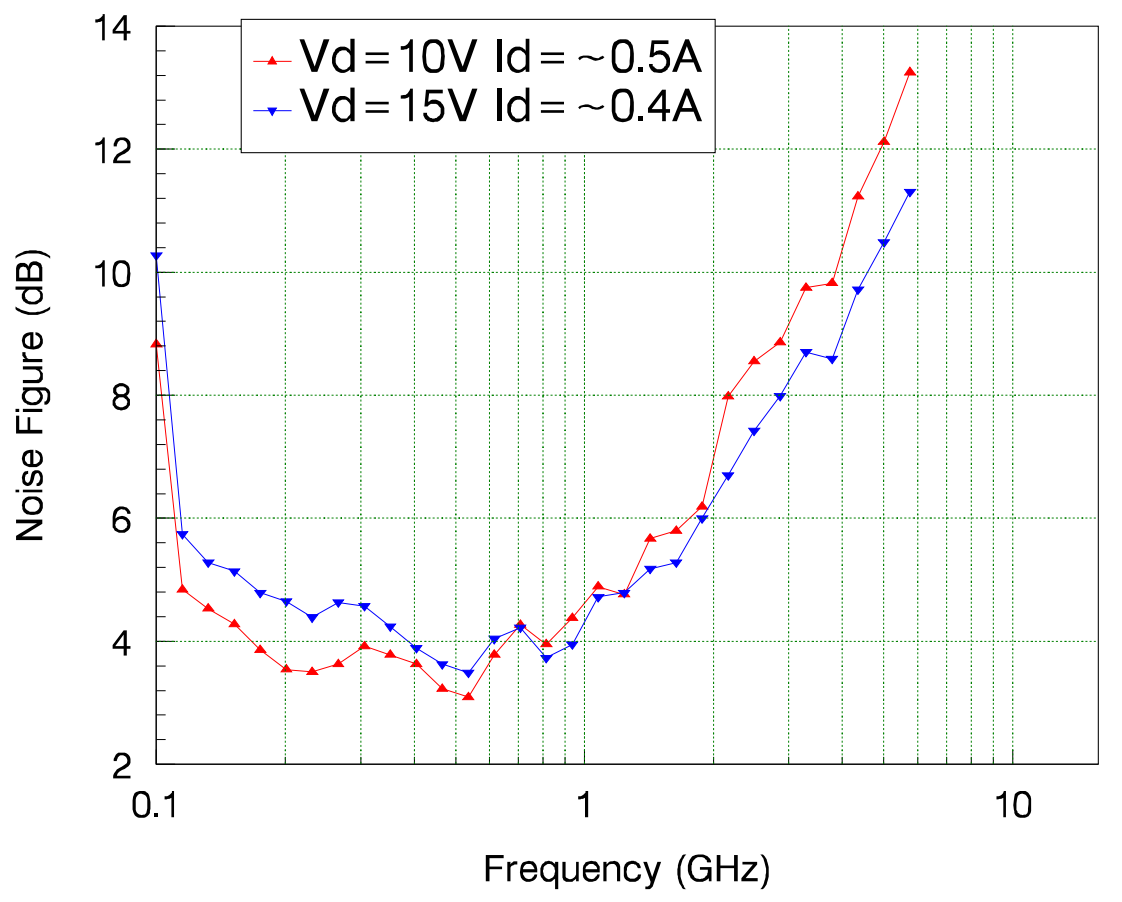

Figure 7. Noise performance of a $1 \mathrm{~mm}$ GaN FET.

In the first instance, we might hope to develop elementary buck converter circuits sufficient for converters operating between $42 \mathrm{~V}$ and $12 \mathrm{~V}$. This range, for example, will be in great demand once domestic automobiles convert to the new standard of $42 \mathrm{~V}$, and a need for systems operating at these levels becomes widespread. This is expected to happen in the 2010-2015 timeframe, leaving little time for us to wait for the reliability to settle before embarking on circuit design, if we are to catch the wave in the automotive market.

\subsection{Vacuum-tube Replacement}

To the amazement of electronic engineers, vacuum tubes continue to be used in three areas today: In highpower microwave amplification in the form of travelling-wave tubes (TWTs), chiefly for satellite application; in very-high power amplification for terrestrial short- and medium-wave long-range transmission; and in high-end sound-studio equipment. GaN has been identified as the likely technology to displace TWTs, and more than one commercial concern has started work on GaN-based, solid-state, travelling-wave, amplifier MMICs. However, using GaN's wide dynamic range to displace vacuum-tubes from the audio sphere has far lower financial incentive, and faces the perception that much vacuum-tube appeal has nothing to do with the electronic performance so much as the history.

We have good scientific reason to expect that the high-voltage capability of GaN combined with its noise performance will give it a wider dynamic range than any previous solid-state technology. ${ }^{11,14,17}$ This is the property of vacuum-tubes that has sustained their use in some applications such as microphone preamplification. ${ }^{16}$ It would be very scientifically interesting to construct simple circuits analogous to the vacuum-tube versions whose dynamic range is crucial, and empirically compare performance.

Some might deem it frivolous to invest such effort in "valve amplifiers". This criticism has some validity. Nevertheless the demonstration will serve to verify an ability of GaN that may have crucial application elsewhere. It also tackles a debate that remains unresolved, and that contributes to the "amazement" of engineers and the popular press over the use of vacuum tubes, and has done so for some decades. Since this application is potentially 
a scientific curiosity and unlikely to blossom into large-volume commercial activity, it is another avenue that can sensibly be pursued without the assurance of reliability. It would be ironic if GaN was to finally displace the vacuum tube in applications such as microphone preamplification after the time that that technology has held onto that niche.

\subsection{Linear Amplifiers}

Debate continues around the question of whether GaN FETs will enable the construction of amplifiers that are intrinsically more linear for a given power-added efficiency (PAE) than silicon. It is acknowledged that silicon, in the form of LDMOS devices, will not be able to provide the required power-distortion performance in base station amplifiers for cellular systems employing complex-modulation schemes, once user frequencies pass the current $2.5 \mathrm{GHz}$ standard. GaN is recognised as a contender for the job, based on its higher operating voltages that imply reduced matching complexity in the power regime in question (tens to hundreds of Watts).

Our speculation is that GaN may be able to offer intrinsically lower distortion than previous technologies, purely through a shift to higher voltages (thus lower currents) and improved thermal properties of substrates. If so, this would suggest that GaN circuits might offer advantages in the regime currently satisfied by silicon, even below $2 \mathrm{GHz}$. The fundamental understanding of the design constraints on power-amplifier distortion in relation to the device characteristics would help designers to assess GaN as an alternative. This is a question not so much of designing a circuit that hits certain performance criteria - the goal of existing PA design work employing GaN devices - but of understanding some fundamentals of power amplifier operation. This investigation is once again suitable for pursuit without waiting for the resolution of reliability details.

\section{CONCLUSION}

We would conclude by identifying the following key understandings:

- GaN is here, and GaN transistors already show very impressive performance;

- GaN has come on-stream quickly, and now is the time to start developing circuit designs in order not to be left behind, concerns of reliability notwithstanding;

- Quite novel applications are possible, and GaN can be expected to forge unique markets.

\section{REFERENCES}

1. Eastman, L.F.; Mishra, U.K.; "The toughest transistor yet [GaN transistors]", IEEE Spectrum, Volume 39, Issue 5, May 2002 Pp28-33.

2. Compound Semiconductor, vol. 12, no. 6, July 2006. [Special issue on GaN Electronics and the wide-bandgap sector.]

3. Strid, E.W.; Gleason, K.R.; "A DC-12 GHz Monolithic GaAsFET Distributed Amplifier", IEEE Transactions on Microwave Theory and Techniques, Vol 82, no. 7, Jul 1982 pp969-975 .

4. Piner, E.L. Singhal, S. Rajagopal, P. Therrien, R. Roberts, J.C. Li, T. Hanson, A.W. Johnson, J.W. Kizilyalli, I.C. Linthicum, K.J., "Device Degradation Phenomena in GaN HFET Technology: Status, Mechanisms, and Opportunities", International Electron Devices Meeting, 2006, 11-13 Dec., 2006 pp1-4.

5. Report on Australian Startup "BluGlass", Compound Semiconductor, vol. 12, no. 10, November 2006, pp1213.

6. "UK Defence Chiefs Feel the Need for GaN", Compound Semiconductor, vol. 12, no. 10, November 2006 , p5.

7. R. J. Trew, "Wide bandgap transistor amplifiers for improved performance microwave power and radar applications", 15th International Conference on Microwaves, Radar and Wireless Communications, MIKON2004, Volume 1, 17-19 May 2004, pp18-23.

8. T. Kikkawa, "Recent progress and future prospects of GaN HEMTs for base-station applications", IEEE Compound Semiconductor Integrated Circuit Symposium, 24-27 October 2004, pp17-20. 
9. R.T. Kemerley, H.B. Wallace and M.N. Yoder, "Impact of wide bandgap microwave devices on DoD systems", Proceedings of the IEEE Volume 90, no. 6, June 2002, pp1059-1064.

10. U. K. Mishra, P. Parikh, and Yi-Feng Wu, "AlGaN/GaN HEMTs-an overview of device operation and applications", Proceedings of the IEEE Volume 90, no. 6, June 2002, pp1022-1031. [This issue concentrated on wide-bandgap technology.]

11. U. K. Mishra, "Gallium nitride electronics: Watt is the limit? [summary of GaN semiconductor devices]", Digest of the 62 $2^{\text {nd }}$ Device Research Conference, 21-23 June, 2004, Vol 1, pp3-5.

12. G. Crupi, D. Xiao, D.M.M.-P. Schreurs, E. Limiti, A. Caddemi, W. De Raedt, and M. Germain, "Accurate Multibias Equivalent-Circuit Extraction for GaN HEMTs", IEEE Transactions on Microwave Theory and Techniques, Volume 54, no. 10, Oct 2006, pp3616-3622.

13. A. Jarndal, B. Bunz, and G. Kompa, "Accurate Large-Signal Modeling of AlGaN-GaN HEMT Including Trapping and Self-Heating Induced Dispersion", 2006 IEEE International Symposium on Power Semiconductor Devices and ICs, 4-8 June 2006, pp1-4.

14. Sungjae Lee, K. J. Webb, V. Tilak, and L. F. Eastman, "Intrinsic noise equivalent-circuit parameters for AlGaN/GaN HEMTs", IEEE Transactions on Microwave Theory and Techniques, Volume 51, no. 5, May 2003, pp1567-1577.

15. Sungjae Lee and K. J. Webb, "The influence of transistor nonlinearities on noise properties", IEEE Transactions on Microwave Theory and Techniques, Volume 53, no. 4, April 2005, pp1314-1321.

16. Sony C800GPAC Studio Tube Condenser Microphone Product information: http://bssc.sel. sony .com/BroadcastandBusiness/DisplayModel?id=24120

17. C. Fazi and P. G. Neudeck, "Wide dynamic range RF mixers using wide-bandgap semiconductors", IEEE MTT-S International Microwave Symposium Digest, Colorado, 8-13 June 1997, vol. 1, pp49-51.

18. R. J. Trew, "SiC and GaN transistors - is there one winner for microwave power applications?", Proceedings of the IEEE Volume 90, no. 6, June 2002, pp1032-1047.

19. S.C. Binari, P.B. Klein, and T.E. Kazior, "Trapping effects in wide-bandgap microwave FETs", 2002 IEEE MTT-S International Microwave Symposium Digest, Vol. 3, 2-7 June 2002, pp1823-1826.

20. M.K. Kazimierczuk, and J. Jozwik, "DC/DC converter with class E zero-voltage-switching inverter andclass E zero-current-switching rectifier", IEEE Transactions on Circuits and Systems, Volume 36, no. 11, Nov 1989, pp1485-1488.

21. Hiroyuki Hase, Hiroo Sekiya, Jianming Lu, and Takashi Yahagi, "Resonant DC-DC Converter with Class-E Oscillator", IEEE Transactions on Circuits and Systems -I: Regular Papers, vol. 53, no. 9, September 2006, pp2025-2035.

22. R.D. Nevels, G.D. Arndt, G.W. Raffoul, J.R. Carl, and A. Pacifico, "Microwave catheter design", IEEE Transactions on Biomedical Engineering, Volume 45, no. 7, July 1998, pp885-890.

23. I. Longo, G.B. Gentili, M. Cerretelli, and N. Tosoratti, "A coaxial antenna with miniaturized choke for minimally invasive interstitial heating", IEEE Transactions on Biomedical Engineering, Volume 50, no. 1, Jan 2003, pp82-88.

24. Heng-Mao Chiu, A.S. Mohan, A.R. Weily, D.J.R. Guy, and D.L. Ross, "Analysis of a novel expanded tip wire (ETW) antenna for microwave ablation of cardiac arrhythmias", IEEE Transactions on Biomedical Engineering, Volume 50, no. 7, July 2003, pp890-899.

25. James C. Lin, "Microwave Surgery Inside the Heart", IEEE Microwave Magazine, Volume 7, number 6, June 2006, pp32-36.

26. Wang Li-li, Ke Xi-zheng, and Xi Xiao-li, "Technique allows simple design of microwave DROs", IEEE International Symposium on Microwave, Antenna, Propagation and EMC Technologies for Wireless Communications, Volume 2, 8-12 Aug 2005, pp944-948. 